\title{
Advances of single-cell genomics and epigenomics in human disease: where are we now?
}

\author{
Rizqah Kamies $^{1}$ (D) . Celia P. Martinez-Jimenez ${ }^{1}$ (D)
}

Received: 6 January 2020 / Accepted: 28 March 2020 / Published online: 8 April 2020

(c) The Author(s) 2020

\begin{abstract}
Cellular heterogeneity is revolutionizing the way to study, monitor and dissect complex diseases. This has been possible with the technological and computational advances associated to single-cell genomics and epigenomics. Deeper understanding of cell-to-cell variation and its impact on tissue function will open new avenues for early disease detection, accurate diagnosis and personalized treatments, all together leading to the next generation of health care. This review focuses on the recent discoveries that single-cell genomics and epigenomics have facilitated in the context of human health. It highlights the potential of single-cell omics to further advance the development of personalized treatments and precision medicine in cancer, diabetes and chronic age-related diseases. The promise of single-cell technologies to generate new insights about the differences in function between individual cells is just emerging, and it is paving the way for identifying biomarkers and novel therapeutic targets to tackle age, complex diseases and understand the effect of life style interventions and environmental factors.
\end{abstract}

\section{Introduction}

Recently, efforts have been made to highlight the importance of moving translational genomic findings to the clinic for the overall improvement of human health (Cho et al. 2016; Regev et al. 2017; Zeggini et al. 2019). Accordingly, these would include the translation of, but are not limited to the experimental discovery of results, the analysis and functional interpretation of results, the generation of large-scale data and the utilization of advanced computational software to handle result output and lastly, the application of result findings in a clinical setting (Behjati et al. 2018; Haghverdi et al. 2016; Zeggini et al. 2019). These applications, in combination with the approval of multiple other ethical, legal, social, economic and political factors could be used to ultimately combat disease, detect early onsets of disease, monitor disease progression and potentially facilitate preventative treatments (Behjati et al. 2018; Gomes et al. 2019; Regev et al. 2017; Zeggini et al. 2019). Although this approach has been successfully applied in some monogenic disorders and

Celia P. Martinez-Jimenez

celia.martinez@helmholtz-muenchen.de

Rizqah Kamies

rizqah.kamies@helmholtz-muenchen.de

1 Helmholtz Pioneer Campus, Helmholtz Zentrum München, 85764 Neuherberg, Germany in rare disease cases where precision medicine techniques are used as a specific or preventative treatment (June et al. 2018; Karczewski and Snyder 2018; Zeggini et al. 2019), the implementation of this comprehensive translational genomics approach to complex chronic diseases in humans is yet to be achieved (Grouse 2015; Regev et al. 2017).

While the analysis of multiple "omic" (genomic, transcriptomic, proteomic and metabolomic) molecular profiles in bulk have been well established to study cellular homeostasis and disruptions as a consequence of disease (Hasin et al. 2017; Karczewski and Snyder 2018; Sun and Hu 2016), most genetic and epigenetic mechanisms are yet to be probed with single-cell resolution. To understand the finer details at the level of a singular cell, sophisticated genomic and epigenomic next-generation sequencing (NGS) technologies have increased the potential for research output immensely (see Clark et al. 2018; Clark et al. 2016; Kelsey et al. 2017; Macaulay et al. 2017; Stuart and Satija 2019). These would include whole-genome profiling techniques of RNA, DNA, proteins, epigenetic modifications, chromatin accessibility and chromosome conformations on the level of an individual cell (described in Clark et al. 2016; Kelsey et al. 2017; Macaulay et al. 2017; Mincarelli et al. 2018; Nagano et al. 2017; Svensson et al. 2018; Wagner et al. 2016). In this review, we will provide a concise description of the impact of single-cell technologies in the context of human health and disease, while technical development and computational 
analysis required for the near-future translational applications of the single-cell genomic discoveries are reviewed elsewhere (see Birnbaum 2018; Luecken and Theis 2019; Song et al. 2019; Tang et al. 2019; Wang and Song 2017). The harmonization and standardization of single-cell technologies will lead to unprecedented discoveries and translational applications from bench to bed (Shalek and Benson 2017; Strzelecka et al. 2018; Wang and Song 2017).

\section{The individuality of cells}

Epigenetic programs are decisive for cell fate decisions, cell identity and cell state (Borsos and Torres-Padilla 2016; Fischer et al. 2019; Trapnell 2015). When RNA transcripts and components of the epitranscriptome initiate a cascade of events in cells, in response to extrinsic or intrinsic stimuli, single-cell genomics and epigenomics can be used to effectively quantify and monitor those dynamic or discrete changes (Clark et al. 2018; Goldman et al. 2019; Mincarelli et al. 2018; Tritschler et al. 2019). This approach is especially important in a seemingly homogenous population of cells, where in most cases, cells are isolated from the same tissue and epigenomic signatures underlying disease are often concealed in bulk samples (Kelsey et al. 2017; Strzelecka et al. 2018; Tritschler et al. 2019; Wang et al. 2018). Additionally, distinguishing the precise intercellular differences is challenging when considering thousands of cells simultaneously. Often, only the most frequent or the most abundant molecular feature is the one detected on average in a given cell population. (Goldman et al. 2019; Haghverdi et al. 2016; Trapnell 2015). Although cellular heterogeneity is essential to the survival of a population, where increased diversity in cells allows increased adaptation to changes in the surrounding milieu (Goldman et al. 2019), increases in cell-to-cell variability have also been associated to age and age-related diseases (Enge et al. 2017; Hernando-Herraez et al. 2019; Martinez-Jimenez et al. 2017). Moreover, a deep understanding of cellular variability and the impact of this variability in tissue function will allow us to understand how changes in cellular dynamics can influence the entire organism and even lead to cancer, diabetes, metabolic disorders and accelerated ageing (Cheung et al. 2018; Ecker et al. 2018; Enge et al. 2017; Hurria et al. 2016).

To effectively capture and observe the morphological and phenotypical differences of cells in a healthy and diseased state using single-cell approaches, the concept of 'cell identity' should be more concisely understood (see Fig. 1). Although no standardized method for defining 'cell identity' exists (Morris 2019), elucidation on these definitions can be given to make accurate functionalbased assumptions on what changes a cell might undergo until it reaches its final destination or 'cell fate' (Kelsey et al. 2017; Morris 2019; Trapnell 2015; Tritschler et al. 2019). Briefly, cell identity can be deconvolved into a 'cell type' and a 'cell state' within a spatiotemporal manner (Camp et al. 2019; Haghverdi et al. 2016; Treutlein et al. 2014). A cell type or sub-type refers to an observable, functional change within a population where properties vary distinctly in response to extrinsic factors, while a cell state refers to a dynamic change that alters the phenotype and function of the cell in a continuous manner and is often regulated intrinsically (see Camp et al. 2019; Chen et al. 2018; Mincarelli et al. 2018; Morris 2019; Trapnell 2015). For instance, the hematopoietic stem cell (HSC) is a well-elaborated example, where a cell type has

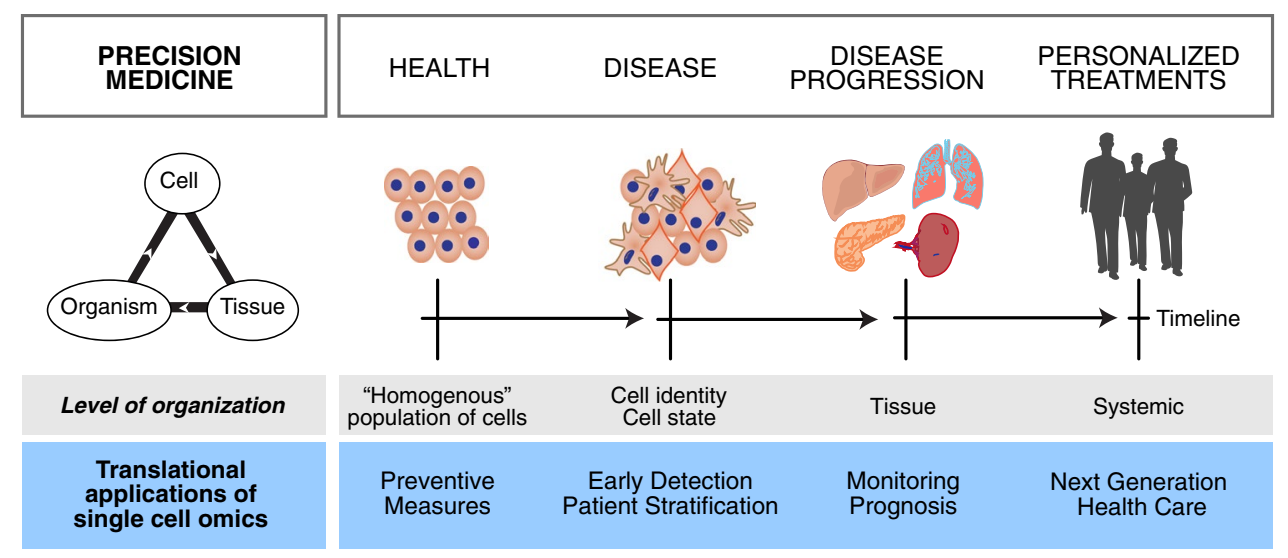

Fig. 1 Single-cell omics is advancing the development of personalized treatments. Precision medicine utilizes genetic information from all levels of cellular organization (cell, tissue, organism) obtained from patient data, to tailor treatments. These novel technologies investigate how cells from a healthy, seemingly homogenous popu- lation of cells can lead to a population with different cellular states, triggering tissue dysfunction and systemic effects (highlighted in grey). The translational applications of single-cell omics will impact on preventive measures, early detection and disease monitoring, leading to the next generation of health care (highlighted in blue) 
the ability to undergo various functional states in order to achieve mature blood and immune cell proliferation after transplantation in a continuous manner, through its multilineage potential and the ability to self-renew (Becker et al. 1963; Mincarelli et al. 2018; Velten et al. 2017). In a nutshell, within the context of health and disease, if we know where a cell comes from, its inherent function and how these functions and states change accordingly to various stimuli, we could predict its fate, with the goal of anticipating the development of a disease or monitoring its progression and outcome (Fig. 1).

These thoughts are echoed by a consortium of researchers who have come together to generate a Human Cell Atlas (Regev et al. 2017), which is an initiative to identify and map all the different cell types in the human body (much like an atlas) for referral of healthy and diseased cells, tissues, organs and systems (Regev et al. 2017; Rozenblatt-Rosen et al. 2017; Strzelecka et al. 2018). The project aims to have a comprehensive composition of all the cell types and identities in the human body, allowing the identification of patterns and interactions at various levels of resolution and to facilitate a functional starting point for researchers when trying to answer relevant health-related questions (Regev et al. 2017; RozenblattRosen et al. 2017). Subsequently, the next step moving forward with a fully annotated and functional human cell atlas would be to concentrate on reducing disease damages, develop methods of preventative treatment, improve disease diagnostics and advance personalized medicine (Strzelecka et al. 2018). Accordingly, these goals are aligned with those of the LifeTime Initiative (https://lifet ime-fetflagship.eu/), a unified research initiative to understand the cause and biological mechanisms behind disease, monitor and track disease changes and progression and ultimately, treat individual human cells affected by disease.

Thus far, such progressive atlas initiatives have been achieved in the model organism Mus musculus through an initiative of the Tabula Muris Consortium (Tabula Muris et al. 2018), where information from a transcriptomic analysis of more than 100000 cells collected from 20 organs and tissues was collated to establish a foundation for an atlas in mouse (Tabula Muris et al. 2018). In humans, the effect would be far more complex as each and every person is different and each and every organ or tissue is affected differently in diseased situations. Furthermore, for complex chronic diseases known to affect human health and expedite the ageing process, single-cell genomic and epigenomic techniques have become necessary for early disease detection, accurate diagnosis and prognosis, monitoring disease progression in tissues and systemically, to facilitate personalized treatment and achieve next-generation health care (see Fig. 1). Here we review recent key findings.

\section{The influence of single-cell approaches}

\section{Cancer}

To date, tumour-biology studies have represented one of the biggest challenges in improving targeted cancer therapies (Levitin et al. 2018; Strzelecka et al. 2018). Moreover, because tumours are an elaborate, complex mix of different cell types and states arising from a single cell that has progressed and diversified through somatic mutations to form distinct subpopulations (Qian et al. 2017; Sierant and Choi 2018; Suvà and Tirosh 2019), intercellular heterogeneity is critical for both accurate diagnosis and personalized treatment (Qian et al. 2017; Sant et al. 2017). The accurate profiling of cellular variability at the single-cell level within a tumour environment where both malignant and immune cells are present (amongst others) could locate the correct gene sequences (biomarkers) for targeted treatment and improve treatment efficiency, specifically through administration of the appropriate drugs to prevent or reduce cancer relapse (Qian et al. 2017; Sierant and Choi 2018; Wagner et al. 2019). This is especially important when only a specific part of the tumour is targeted and proliferation is still possible even from a minute proportion of cells, often concealed in bulk analyses (Qian et al. 2017; Tirosh and Suvà 2019). For example, in studies investigating lung adenocarcinomas, accurate profiling of tumours for targeted treatment remains challenging due to increased tumour heterogeneity (Zhang et al. 2019). While mutations of the epidermal growth factor receptor $(E F G R)$ in non-small cell lung cancers (NSCLC) have been widely employed as biomarkers for lung carcinogenesis (Harrison et al. 2019; Zhang et al. 2019), resistance to the well-established EGFR tyrosine kinase inhibitors has been frequently reported, most likely due to $E G F R$ T790M resistance mutations (Del Re et al. 2018; Rexer et al. 2009; Sullivan and Planchard 2016). Although classical histomorphologic evaluation of a malignant tumour is argued to be the most significant diagnostic, prognostic and predictive biomarker with the greatest impact on patient treatment (Radpour and Forouharkhou 2018), indepth single-cell RNA sequencing in combination with protein profiling of primary human tumours has been proposed to be highly effective in discerning cell types and subtypes within tumour cells and for detection of distinct functional states of proliferation (Tirosh and Suvà 2019).

To elaborate, an integrated analysis of cancer cells has been shown in hepatocellular carcinoma (HCC) where time-of-flight mass cytometry (CyTOF) and single-cell RNA sequencing were used to show an immunosuppressive gradient of immune cells in a tumour microenvironment, non-tumour microenvironment and peripheral 
blood with the goal of describing the phenotypical characteristics of the T-cell subsets (Chew et al. 2017). The authors describe the enrichment of regulatory T-cells, $\mathrm{CD} 8+\mathrm{T}$-cells and natural killer cells in the tumour microenvironment in conjunction with the expression of multiple markers for T-cell exhaustion, when compared to T-cell subsets in the non-tumour microenvironment (Chew et al. 2017). In high-grade serous ovarian cancer (HGSOC), CyTOF was employed with un-supervised computational analysis for an in-depth phenotypical characterization of both dominant and rare cell phenotypes linked to surface markers, intracellular signalling proteins, transcription factors and cell-cycle proteins active in the malignancy (Gonzalez et al. 2018). Recently, a pilot study involving human bone marrow cells has been subjected to combinatorial single-cell RNA sequencing, multiparameter flow cytometry and mass cytometry to show diversity across human samples over the full range of adulthood and to act as a reference for different cell populations (Oetjen et al. 2018).

For further investigation of solid tumours, the invasive human epidermal growth factor 2 (HER2) protein part of the ERBB pathway is active in numerous cancers including pancreatic, ovarian, breast, gastric, lung, glioma/glioblastoma, colorectal, in the central nervous system, and is one of the most well-studied oncogenes (Masoud and Pagès 2017; Townsend et al. 2018). In particular, the cancer-related HER 2 mutation is often used as a biomarker for $15-20 \%$ of all breast cancer tumours (Rye et al. 2018) and has been successfully targeted for gene therapy (Chung et al. 2017; Masoud and Pagès 2017). Moreover, the oncogene has been extensively studied with single-cell RNA sequencing, particularly to show extensive intratumoral heterogeneity (Cho 2019; Chung et al. 2017; Wang et al. 2019a, b). Recently, Cho (2019) showed triple-negative breast cell populations identified by three subtyping marker genes (ERBB2 also known as HER2, ESRI and $P G$ ), while immune landscape cell populations, consisting of subclasses of both tumour and non-tumour (immune) cells, were shaped by distinctive gene expression signatures inferred from copy number alterations within the tumour microenvironment by Chung et al. (2017). Subsequent single-cell RNA sequencing investigations of HER2+ with the monoclonal antibody, Trastuzumab (Herceptin), successful in targeting HER2+ breast cancer cells (Wang et al. 2019b), re-affirmed previously highlighted gene sequences such as $C L U$ and SEPPI genes in trastuzumabtreated patients and indicated new gene signatures of interest such as the chemokine ligands $C X C L 1$ and $C X C L 8$ that were significantly downregulated under trastuzumab treatment (Wang et al. 2019a). In addition, further single-cell RNA sequencing of trastuzumab resistance patients has also been performed (Wang et al. 2019b), where studies have shown that perhaps a combination of inhibitors targeting
CDK4/6 inhibitor-resistant tumours are required, specifically those targeting a immunosuppressive immature myeloid cell (IMC) population in resistant tumours (Wang et al. 2019b).

The KRAS mutation has been reported to be the most frequently mutated oncogene in human tumours and has been the investigative target in many cancer studies specifically in colorectal, pancreatic and in non-small lung cancers (Kim et al. 2018; Kuboki et al. 2019; Roerink et al. 2018; Román et al. 2018). The extensive mutation rate of the KRAS oncogene has played detrimental roles in cancer initiation, propagation and maintenance and thus could be highlighted as a therapeutic target for specific treatment (Cox et al. 2014; Kim et al. 2018). Recently, the CRISPR-Cas9 system has been employed as a proof of concept study, where guide RNAs specific to targeted gene sequences present on mutant $K R A S$ alleles were employed with the goal of removing gene sequences known to cause malignancies (Kim et al. 2018). Although, the study proved successful in targeting mutant gene sequences for manipulation, the authors conclude that the technique alone is not enough to induce tumour remission, but could be considered as a gene therapy to reduce tumour volume by blocking tumour growth in vivo before surgery (Kim et al. 2018).

The literature reported on targeted cancer biomarkers thus far is considerable, many of which include Chimeric Antigen Receptor (CAR) T-cell therapy that have been successful or have been making progress in personalized cancer treatment (see Townsend et al. 2018). These genetically engineered CAR T-cells have shown immunotherapy action against numerous haematological malignancies, some of which include the famous CD19 protein for treatment of acute lymphoblastic leukaemia and large B-cell lymphoma (Feins et al. 2019) and new receptor targets (CD5, CD123, CD33, CD70, CD38, and BCMA) that are currently being evaluated and have already shown positive results (Townsend et al. 2018).

\section{Diabetes}

The main source of diabetes development and disease progression has been attributed to disturbances in the regulation and synchronization of the hormone-producing cells in the pancreatic islets (Carrano et al. 2017). These cells are clusters of at least five different endocrine cell types (alpha, beta, delta, gamma and epsilon) that each produce a unique hormone to function together in a well-orchestrated manner in controlling and maintaining blood glucose levels (Da Silva Xavier 2018). While alpha and beta endocrine cells are stimulated to release glucagon and insulin, respectively (Da Silva Xavier 2018; Theis and Lickert 2019), discordance in both cell types has been shown to increase disease pathogenesis in type 1 and type 2 diabetes (Ackermann et al. 2016; 
Brissova et al. 2018; Tritschler et al. 2017). Moreover, an increase in hyperglycaemia has been associated with a loss of beta-cell mass, function and organization and is the cell type most frequently studied for insulin resistance (Carrano et al. 2017; Lawlor et al. 2017b; Segerstolpe et al. 2016; Theis and Lickert 2019; Tritschler et al. 2017).

Notably, single-cell transcriptome profiling has been utilized in the past few years to discern cellular heterogeneity within the islets of Langerhans (Fischer et al. 2019; Tritschler et al. 2019, 2017), particularly for beta cells (Baron et al. 2016; Lawlor et al. 2017a; Segerstolpe et al. 2016; Teo et al. 2018; Xin et al. 2016). Segerstolpe et al. (2016) investigated cell-type specific gene expression in the pancreas of healthy and type 2 diabetic individuals and uncovered major gene expression differences (transcriptional signatures) between exocrine and endocrine cell types, including the less abundant cell types such as human delta, gamma and epsilon cells. Previously, these cells had been difficult to observe due to bulk characterization methods (Lawlor et al. 2017a), however, single-cell RNA sequencing has shed light on the novel roles for each rare cell type based on their activated signalling pathways and receptor proteins (Lawlor et al. 2017a; Segerstolpe et al. 2016). For example, insight into the transcriptome of the minority cell type, epsilon cells and its ghrelin-producing capability was provided (Segerstolpe et al. 2016), as well as the expression of the rare delta and gamma cell types that are prompted by hormonal cues from leptin, ghrelin and dopamine signalling pathways to facilitate metabolic signalling in the pancreas (Lawlor et al. 2017a). Further single-cell RNA investigations by Xin et al. (2016) showed a total of 245 genes to be affected by type 2 diabetes when compared to non-diabetic single-cell transcriptomes. Among the common transcript expression profiles found between the human islet cells, only 20 genes (for example, RBP4, DLK1, ADCYAP1, RGS16, SOX4, BMP5, TIMP2, TSPAN1, MAFB and TFF3) were specific to a certain cell type (Xin et al. 2016). Lastly, a few recent reviews have tracked the progress of genes linked to specific endocrine cell types in these studies (see Chiou et al. 2019; Tritschler et al. 2017), with some going as far as to re-analyse the single-cell transcriptome datasets using a machine learning approach (Ma and Zheng 2018). The in-depth analyses reported on oxidative stress being the perpetrator to enhance beta-cell dysfunction as a final result, together with the potential activation of pathways linked to beta-cell apoptosis that may be the resulting cause of an insulin gene expression deficit in type 2 diabetes (Ma and Zheng 2018).

Furthermore, there has been a notion that alpha cells have the ability to transdifferentiate into beta cells under conditions of extreme metabolic tress or when prompted under strong metabolic signalling (Ackermann et al. 2016; Tritschler et al. 2017). This has been postulated to be in part, due to the flexibility in the epigenome of alpha cells (Ackermann et al. 2016), where multiple bivalent activating and repressing histone marks (H3K4me3 and H3K27me, respectively) on gene loci associated with alpha and beta cells have been identified (Ackermann et al. 2016; Bramswig et al. 2013). In addition, more areas of open chromatin $(\sim 75 \%)$ in alpha cells, in comparison to beta cells have been detected, many of which were associated with beta-cell signature genes (Ackermann et al. 2016; Tritschler et al. 2017). Recently, efforts have been made to observe cell-type specific transcriptomes mapped to areas of open chromatin to define gene regulatory regions, characterize novel gene signatures and highlight transcription factors of interest pertaining to diabetes pathogenesis (Ackermann et al. 2016; Bysani et al. 2019; Chiou et al. 2019; Rai et al. 2019). Most of these studies have used the Assay for Transposase Accessible Chromatin Sequencing (ATAC-seq) technique (Buenrostro et al. 2015; Lareau et al. 2019) for profiling rare and common endocrine cell types.

An intriguing study by Chiou et al. (2019) used a sophisticated new approach to obtain ATAC-seq profiles from single nuclei (snATAC-seq) to show differentiated regions of open chromatin from heterogenous cell types and subtypes with the aim of highlighting molecular mechanisms linked to genetic risk variants of type 2 diabetes. The authors were able to localize 239 fine-mapped type 2 diabetes risk signals to areas of open chromatin and ordered variants in islets at these signals with predicted regulatory functions to known target genes such as the $K C N Q 1$ locus (Chiou et al. 2019). Further insight into how type 2 diabetes alters chromatin organization and allows the subsequent affinity for suitable transcription factors and thus gene expression in pancreatic islets was provided by Bysani et al. (2019). A total of 1078 regions of open chromatin corresponding to 898 genes were detected and differentially expressed between diabetic and non-diabetic islets, many of which were annotated to genes linked to islet dysfunction and type 2 diabetes instigators such as HHEX, HMGA2, GLIS3, MTNR1B, PARK2 and some previously associated single-nucleotide polymorphisms (SNPs) (Bysani et al. 2019). Furthermore, a large proportion of ATAC-seq peaks were mapped near to transcription start sites for easy manipulation by cis-regulatory elements, particularly enhancers and in areas where celltype specific transcription factors such as FOXA, MAFB, NKX2.2, NKX6.1 and PDX1 for type 2 diabetes bind (Bysani et al. 2019). It is important to note that the overall goal of observing cell-type expression profiles contributing to type 2 diabetes is to reveal novel druggable targets for pathway manipulation and further approaches to prevent, monitor and treat type 2 diabetes (Chiou et al. 2019; Lawlor et al. 2017a; Tritschler et al. 2017). 


\section{Chronic and age-related diseases}

Ageing can be defined as the progressive decline in physiological and cellular functions (Enge et al. 2017; López-Otín et al. 2013). Although ageing has not been classified as a disease by the World Health Organization (WHO), it is considered a leading risk factor for all chronic diseases (WHO 2009). From epidemiological studies and experimental data, we know that chronic diseases accelerate the ageing process, suggesting that ageing and chronic diseases share common molecular mechanisms (Kennedy et al. 2014). For instance, the incidence of cancer, diabetes, kidney disease (O'Sullivan et al. 2017; Rowland et al. 2018) and non-alcoholic fatty liver disease (NAFLD) (Hunt et al. 2019; Ogrodnik et al. 2017) increases with age. Age-related disease in the kidney has been linked to nephrosclerosis, impaired renal function and chronic kidney disease (O'Sullivan et al. 2017; Rowland et al. 2018). In the liver, NAFLD is the most common chronic liver disease and comprises a range of related disorders in which the earliest stage is the accumulation of lipids in the liver followed by steatohepatitis that is generally marked by liver inflammation. Later on, steatohepatitis may progress to liver fibrosis and liver failure. Non-alcoholic fatty liver and steatohepatitis are reversible in their early stages, but ultimately may lead to the development of cirrhosis (that is an irreversible state) and hepatocellular carcinoma (HCC) (Podrini et al. 2013).

Recently, single-cell RNA sequencing has been used to study the complex cellular architecture of the kidney and investigate how changes in gene expression patterns are associated with chronic kidney disease (Chen et al. 2017; Der et al. 2017; Lake et al. 2019; Liao et al. 2020; Park et al. 2019,2018 ). The human kidney is a highly complex tissue comprised of at least 30 different cell types that function in an intricate filtration system to remove nitrogen, water and other waste products from the blood, maintain electrolyte balance and red blood cell production and regulate blood pressure through hormonal secretion (Lake et al. 2019; Park et al. 2018; Rowland et al. 2019). Park et al. (2018) profiled approximately 58,000 cells isolated from heathy mouse kidneys and discovered that 21 homologous genes in humans were associated with monogenic inheritance of proteinuria and other complex-trait diseases such as chronic kidney disease and nephrolithiasis (Park et al. 2018). Similarly, by means of single-nucleus RNA sequencing (snRNA-seq), Lake et al. (2019) have shown the power of this technology to analyse clinical samples bypassing technical limitations in the enzymatic dissociation of the solid tissue and using limiting amounts of sample (Lake et al. 2019). Further analysis of receptor-ligand signalling pathways among cell types showed how the dysregulation of expression profiles associated to integrins in multiple cell types play a major role in the development of human kidney disease (Lake et al. 2019).
Likewise, single-cell genomics has emphasized the cellular heterogeneity present in the liver, with regard to the liver zonation of hepatocytes (Aizarani et al. 2019; Dobie et al. 2019; Halpern et al. 2018, 2017; Ramachandran et al. 2019) and the variability among non-parenchymal cells during chronic liver disease (Krenkel et al. 2020; MacParland et al. 2018; Pepe-Mooney et al. 2019; Su et al. 2017; Xiong et al. 2019). MacParland et al. (2018) identified 20 distinct cell populations of hepatocytes, endothelial cells, cholangiocytes, hepatic stellate cells and resident cells from the immune compartment. Beyond a liver cell atlas, this work identified two different populations of intrahepatic $\mathrm{CD} 68^{+}$ macrophage populations with inflammatory or immunoregulatory properties, respectively (MacParland et al. 2018). Later on, Aizarani et al. (2019) sequenced CD45 ${ }^{+}$ and $\mathrm{CD}_{45^{-}}$cells isolated from hepatocellular carcinomas from three patients showing how the gene expression signatures and biomarkers of liver cell types can be monitored in human liver disease.

Other chronic liver diseases such as Non-Alcoholic SteatoHepatitis (NASH) and liver fibrosis have also been investigated at the single-cell level (Dobie et al. 2019; Ramachandran et al. 2019; Xiong et al. 2019). The intercellular signalling between non-parenchymal cells (endothelial cells, Kuppfer cells and cholangiocytes) was analysed with single-cell transcriptomics and secretome analysis to reveal intercellular cross-talk via ligand and receptor signalling, in diet-induced NASH mice livers (Xiong et al. 2019). Comparative studies between mouse and human revealed a highly conserved pattern among liver cell types across species (Xiong et al. 2019). In addition, a novel NASH-specific macrophage population (termed NAM) was identified, suggesting that NASH alters the functional properties of liver macrophages populations by increasing Trem 2 protein levels in a subset of macrophages, therefore increasing liver heterogeneity during pathogenesis of NASH (Xiong et al. 2019).

Recently, more than 100,000 cells isolated from healthy and cirrhotic human livers were analysed by single-cell RNA sequencing to further characterize the fibrotic niche and the cross-talk between non-parenchymal cells in the liver (Ramachandran et al. 2019). Ramachandran et al. (2019) have identified a scar-associated TREM $2^{+} \mathrm{CD}^{+}$subpopulation of macrophages that expands in liver fibrosis with a pro-fibrogenic phenotype. Additional endothelial subpopulations characterized by a high expression of PLVAP, CD34 and ACKR1, and mesenchymal cells expressing PDGFRA were also expanded in the pathogenesis of the liver disease (Ramachandran et al. 2019). Moreover, the zonation pattern of hepatic stellate cells across the hepatic lobe was also altered during liver fibrosis (Dobie et al. 2019). In summary, the unbiased analysis of the multi-lineage interactome between healthy and disease-affected livers will uncover novel molecular pathways which are potentially druggable, 
leading us to a new era of precision medicine (Ramachandran et al. 2019).

\section{Single-cell genomics and organoids: emerging diagnostic tool to understand tissue development and human disease}

As the scope for human biological research expands, new personalized in vitro models are emerging as a diagnostic tool to study disease development and progression. These three-dimensional tissue cultures, termed organoids, are initiated from either pluripotent embryonic stem cells, induced pluripotent stem cell counterparts or tissue-resident adult stem cells (Clevers 2016; Huch et al. 2017; Lancaster and Huch 2019). Organoids recapitulate with sufficient complexity, molecular and cellular processes present in the original tissue (Camp and Treutlein 2017; Lancaster and Huch 2019). Therefore, this powerful system replicates in vitro, some level of organ development and disease phenotypes for a wide variety of tissues (Camp et al. 2019, 2018; Clevers 2016; Huch et al. 2017). These attributes make organoid models an ideal system to study complex disease phenotypes and implement personalized therapies in a controlled environment (Camp et al. 2019; Camp and Treutlein 2017). In addition, human organoids are genetically stable, can be long-term expanded in vitro and are composed of a collection of differentiated cell states that mimic the cellular composition in the corresponding original organ (Grassi et al. 2019; Hu et al. 2018; Huch et al. 2017; Lancaster and Huch 2019). For these reasons, the combination of singlecell omics and organoids has become an exceptional in vitro tool to dissect the molecular mechanisms underlying complex human diseases (Camp et al. 2019; Camp and Treutlein 2017).

At present, single-cell genomics has been applied to organoids modelling several human tissues (Brazovskaja et al. 2019) including brain (Camp et al. 2015; Kanton et al. 2019; Tanaka et al. 2020), kidney (Harder et al. 2019), liver (Camp et al. 2017; Huch et al. 2015), lung (Lee et al. 2017; Sachs et al. 2019) and intestine (Mithal et al. 2020). This line of research has advanced our understanding of the molecular pathways involved in the pathogenesis of the disease, for instance in glomerular disease of the kidney (Harder et al. 2019), inflammatory bowel disease in the intestine (Mithal et al. 2020) and respiratory viral infection in the lung (Sachs et al. 2019). In particular, recent studies have shown the translational applications of liver organoids to model hepatic steatosis (Kruitwagen et al. 2017), steatohepatitis (Ouchi et al. 2019), alcohol liver injury (Wang et al. 2019c) and alpha-1 antitrypsin deficiency (Gómez-Mariano et al. 2020). Furthermore, combining organoids with gene editing tools such as the CRISPR/Cas 9 system has opened up a wealth of opportunity, as organoids are highly amendable to genome editing (Fujii et al. 2019). For instance, the application of organoid models in tumour-biology studies has provided a patient-specific functional testing platform for drug administration and sensitivity, as both phenotypic and genomic results can be retrieved, molecular mechanisms can be identified and personalized treatment strategies can be initiated (Clevers 2016; Drost and Clevers 2018; Huch et al. 2017). These pioneering works would potentially allow the identification of biomarkers and further personalized treatments (Huch et al. 2017).

\section{Conclusion}

Single-cell genomic approaches are changing the concept of personalized medicine from early detection to tailored treatments. The harmonization and standardization of singlecell technologies is leading to translational applications from bench to bed. The identification of new and rare cell types in an early stage, the precise monitoring of their molecular changes and their contribution to disease pathogenesis and outcome are key stepping stones for implementing these technologies in the clinical practice.

Still future efforts will be needed to dissect how complex diseases are influenced by lifestyle, dietary interventions and ageing. Single-cell multiomics is emerging as a novel technology to read out multiple layers of genetic and epigenetic information simultaneously, aiming to anticipate changes in cell fate and cellular function. Overall, single-cell genomics is opening a new frontier in the field of personalized medicine leading to the next generation of health care.

Acknowledgments Open Access funding provided by Projekt DEAL. R. Kamies is funded by the Postdoctoral Fellowship Program of the Helmholtz Zentrum München.

\section{Compliance with ethical standards}

Conflict of interest: On behalf of all authors, the corresponding author states that there is no conflict of interest.

Open Access This article is licensed under a Creative Commons Attribution 4.0 International License, which permits use, sharing, adaptation, distribution and reproduction in any medium or format, as long as you give appropriate credit to the original author(s) and the source, provide a link to the Creative Commons licence, and indicate if changes were made. The images or other third party material in this article are included in the article's Creative Commons licence, unless indicated otherwise in a credit line to the material. If material is not included in the article's Creative Commons licence and your intended use is not permitted by statutory regulation or exceeds the permitted use, you will need to obtain permission directly from the copyright holder. To view a copy of this licence, visit http://creativecommons.org/licenses/by/4.0/. 


\section{References}

Ackermann AM, Wang Z, Schug J, Naji A, Kaestner KH (2016) Integration of ATAC-seq and RNA-seq identifies human alpha cell and beta cell signature genes. Mol Metab 5:233-244. https://doi. org/10.1016/j.molmet.2016.01.002

Aizarani N et al (2019) A human liver cell atlas reveals heterogeneity and epithelial progenitors. Nature 572:199-204. https://doi. org/10.1038/s41586-019-1373-2

Baron M et al (2016) A single-cell transcriptomic map of the human and mouse pancreas reveals inter- and intra-cell population structure. Cell Syst 3:346-360. https://doi.org/10.1016/j. cels.2016.08.011

Becker AJ, Mc CE, Till JE (1963) Cytological demonstration of the clonal nature of spleen colonies derived from transplanted mouse marrow cells. Nature 197:452-454. https://doi. org/10.1038/197452a0

Behjati S, Lindsay S, Teichmann SA, Haniffa M (2018) Mapping human development at single-cell resolution. Development 145:dev152561. https://doi.org/10.1242/dev.152561

Birnbaum KD (2018) Power in numbers: single-cell RNA-seq strategies to dissect complex tissues. Annu Rev Genet 52:203-221. https://doi.org/10.1146/annurev-genet-120417-031247

Borsos M, Torres-Padilla ME (2016) Building up the nucleus: nuclear organization in the establishment of totipotency and pluripotency during mammalian development. Genes Dev 30:611-621. https ://doi.org/10.1101/gad.273805.115

Bramswig NC et al (2013) Epigenomic plasticity enables human pancreatic $\alpha$ to $\beta$ cell reprogramming. J Clin Invest 123:1275-1284. https://doi.org/10.1172/JCI66514

Brazovskaja A, Treutlein B, Camp JG (2019) High-throughput singlecell transcriptomics on organoids. Curr Opin Biotechnol 55:167171. https://doi.org/10.1016/j.copbio.2018.11.002

Brissova $\mathrm{M}$ et al (2018) $\alpha$ Cell function and gene expression are compromised in type 1. Diabetes Cell Rep 22:2667-2676. https://doi. org/10.1016/j.celrep.2018.02.032

Buenrostro JD, Wu B, Chang HY, Greenleaf WJ (2015) ATAC-seq: a method for assaying chromatin accessibility genome-wide. Curr Protoc Mol Biol 109:21.29.21-21.29.29. https://doi. org/10.1002/0471142727.mb2129s109

Bysani M et al (2019) ATAC-seq reveals alterations in open chromatin in pancreatic islets from subjects with type 2 diabetes. Sci Rep 9:7785. https://doi.org/10.1038/s41598-019-44076-8

Camp JG et al (2015) Human cerebral organoids recapitulate gene expression programs of fetal neocortex development. Proc Natl Acad Sci USA 112:15672. https://doi.org/10.1073/pnas.15207 60112

Camp JG, Platt R, Treutlein B (2019) Mapping human cell phenotypes to genotypes with single-cell genomics. Science 365:1401. https ://doi.org/10.1126/science.aax6648

Camp JG et al (2017) Multilineage communication regulates human liver bud development from pluripotency. Nature 546:533-538. https://doi.org/10.1038/nature22796

Camp JG, Treutlein B (2017) Human organomics: a fresh approach to understanding human development using single-cell transcriptomics. Development 144:1584. https://doi.org/10.1242/ dev. 150458

Camp JG, Wollny D, Treutlein B (2018) Single-cell genomics to guide human stem cell and tissue engineering. Nat Methods 15:661667. https://doi.org/10.1038/s41592-018-0113-0

Carrano AC, Mulas F, Zeng C, Sander M (2017) Interrogating islets in health and disease with single-cell technologies. Mol Metab 6:991-1001. https://doi.org/10.1016/j.molmet.2017.04.012

Chen L et al (2017) Transcriptomes of major renal collecting duct cell types in mouse identified by single-cell RNA-seq. Proc Natl
Acad Sci USA 114:E9989. https://doi.org/10.1073/pnas.17109 64114

Chen X, Teichmann SA, Meyer KB (2018) From tissues to cell types and back: single-cell gene expression analysis of tissue architecture. Annu Rev Biomed Data Sci 1:29-51. https://doi. org/10.1146/annurev-biodatasci-080917-013452

Cheung P et al (2018) Single-cell chromatin modification profiling reveals increased epigenetic variations with. Aging Cell 173:1385-1397.e1314. https://doi.org/10.1016/j. cell.2018.03.079

Chew V et al (2017) Delineation of an immunosuppressive gradient in hepatocellular carcinoma using high-dimensional proteomic and transcriptomic analyses. Proc Natl Acad Sci USA 114:E5900E5909. https://doi.org/10.1073/pnas.1706559114

Chiou J et al. (2019) Single cell chromatin accessibility reveals pancreatic islet cell type- and state-specific regulatory programs of diabetes risk bioRxiv:693671. https://doi.org/10.1101/693671

Cho SY (2019) Identification of ERBB pathway-activated cells in triple-negative breast cancer. Genomics Inform 17:e3. https:// doi.org/10.5808/GI.2019.17.1.e3

Cho YS, Chung KW, Kim N-S (2016) Translational genomics for human diseases: toward a new era of precision medicine. Genes Genomics 38:573-575. https://doi.org/10.1007/s1325 8-016-0433-3

Chung W et al (2017) Single-cell RNA-seq enables comprehensive tumour and immune cell profiling in primary breast cancer. Nat Commun 8:15081. https://doi.org/10.1038/ncomms15081

Clark SJ et al (2018) scNMT-seq enables joint profiling of chromatin accessibility DNA methylation and transcription in single cells. Nat Commun 9:781. https://doi.org/10.1038/s41467-018-03149 $-4$

Clark SJ, Lee HJ, Smallwood SA, Kelsey G, Reik W (2016) Singlecell epigenomics: powerful new methods for understanding gene regulation and cell identity. Genome Biol 17:72. https:// doi.org/10.1186/s13059-016-0944-x

Clevers H (2016) Modeling development and disease with organoids. Cell 165:1586-1597. https://doi.org/10.1016/j.cell.2016.05.082

Cox AD, Fesik SW, Kimmelman AC, Luo J, Der CJ (2014) Drugging the undruggable RAS: mission possible? Nat Rev Drug Discov 13:828-851. https://doi.org/10.1038/nrd4389

Da Silva XG (2018) The cells of the islets of langerhans. J Clin Med 7:54. https://doi.org/10.3390/jcm7030054

Del Re M et al (2018) Concise review: resistance to tyrosine kinase inhibitors in non-small cell lung cancer: the role of cancer stem cells. Stem Cells 36:633-640. https://doi.org/10.1002/stem.2787

Der E et al (2017) Single cell RNA sequencing to dissect the molecular heterogeneity in lupus nephritis. JCI Insight. https://doi. org/10.1172/jci.insight.93009

Dobie R et al (2019) Single-cell transcriptomics uncovers zonation of function in the mesenchyme during liver fibrosis. Cell Rep 29:1832-1847. https://doi.org/10.1016/j.celrep.2019.10.024

Drost J, Clevers H (2018) Organoids in cancer research. Nat Rev Cancer 18:407-418. https://doi.org/10.1038/s41568-018-0007-6

Ecker S, Pancaldi V, Valencia A, Beck S, Paul DS (2018) Epigenetic and transcriptional variability shape phenotypic plasticity. BioEssays 40:1700148. https://doi.org/10.1002/bies.201700148

Enge M, Arda HE, Mignardi M, Beausang J, Bottino R, Kim SK, Quake SR (2017) Single-cell analysis of human pancreas reveals transcriptional signatures of aging and somatic mutation patterns. Cell 171:321-330. https://doi.org/10.1016/j.cell.2017.09.004

Feins S, Kong W, Williams EF, Milone MC, Fraietta JA (2019) An introduction to chimeric antigen receptor (CAR) T-cell immunotherapy for human cancer. Am J Hematol 94:S3-S9. https:// doi.org/10.1002/ajh. 25418 
Fischer DS et al (2019) Inferring population dynamics from single-cell RNA-sequencing time series data. Nat Biotechnol 37:461-468. https://doi.org/10.1038/s41587-019-0088-0

Fujii M, Clevers H, Sato T (2019) Modeling human digestive diseases with CRISPR-Cas9-modified organoids. Gastroenterology 156:562-576. https://doi.org/10.1053/j.gastro.2018.11.048

Goldman SL, MacKay M, Afshinnekoo E, Melnick AM, Wu S, Mason CE (2019) The impact of heterogeneity on single-cell sequencing. Front Genet 10:8-8. https://doi.org/10.3389/fgene .2019 .00008

Gomes T, Teichmann SA, Talavera-López C (2019) Immunology driven by large-scale single-cell sequencing. Trends Immunol 40:1011-1021. https://doi.org/10.1016/j.it.2019.09.004

Gómez-Mariano G et al (2020) Liver organoids reproduce alpha-1 antitrypsin deficiency-related liver disease. Hepatol Int 14:127-137. https://doi.org/10.1007/s12072-019-10007-y

Gonzalez VD et al (2018) Commonly occurring cell subsets in highgrade serous ovarian tumors identified by single-cell mass cytometry. Cell Rep 22:1875-1888. https://doi.org/10.1016/j. celrep.2018.01.053

Grassi L et al (2019) Organoids as a new model for improving regenerative medicine and cancer personalized therapy in renal diseases. Cell Death Dis 10:201. https://doi.org/10.1038/s4141 9-019-1453-0

Grouse L (2015) Translational genetic research of complex diseases. J Transl Int Med 3:137-143. https://doi.org/10.1515/ jtim-2015-0020

Haghverdi L, Büttner M, Wolf FA, Buettner F, Theis FJ (2016) Diffusion pseudotime robustly reconstructs lineage branching. Nat Methods 13:845-848. https://doi.org/10.1038/nmeth.3971

Halpern KB et al (2018) Paired-cell sequencing enables spatial gene expression mapping of liver endothelial cells. Nat Biotechnol 36:962-970. https://doi.org/10.1038/nbt.4231

Halpern KB et al (2017) Single-cell spatial reconstruction reveals global division of labour in the mammalian liver. Nature 542:352-356. https://doi.org/10.1038/nature21065

Harder JL et al (2019) Organoid single cell profiling identifies a transcriptional signature of glomerular disease. JCI Insight. https:// doi.org/10.1172/jci.insight.122697

Harrison PT, Vyse S, Huang PH (2019) Rare epidermal growth factor receptor (EGFR) mutations in non-small cell lung cancer. Semin Cancer Biol. https://doi.org/10.1016/j.semcancer.2019.09.015

Hasin Y, Seldin M, Lusis A (2017) Multi-omics approaches to disease. Genome Biol 18:83. https://doi.org/10.1186/s13059-017-1215-1

Hernando-Herraez I et al (2019) Ageing affects DNA methylation drift and transcriptional cell-to-cell variability in mouse muscle stem cells. Nat Commun 10:4361. https://doi.org/10.1038/s4146 7-019-12293-4

$\mathrm{Hu} \mathrm{H}$ et al (2018) Long-Term expansion of functional mouse and human hepatocytes as 3D organoids. Cell 175:1591-1606. https ://doi.org/10.1016/j.cell.2018.11.013

Huch $\mathrm{M}$ et al (2015) Long-term culture of genome-stable bipotent stem cells from adult human liver. Cell 160:299-312. https:// doi.org/10.1016/j.cell.2014.11.050

Huch M, Knoblich JA, Lutolf MP, Martinez-Arias A (2017) The hope and the hype of organoid research. Development 144:938. https ://doi.org/10.1242/dev.150201

Hunt NJ, Kang SWS, Lockwood GP, Le Couteur DG, Cogger VC (2019) Hallmarks of aging in the liver. Comput Struct Biotechnol J 17:1151-1161. https://doi.org/10.1016/j.csbj.2019.07.021

Hurria A, Jones L, Muss HB (2016) Cancer treatment as an accelerated aging process: assessment, biomarkers, and interventions. Am Soc Clin Oncol Educ Book. https://doi.org/10.1200/ EDBK_156160
June CH, O’Connor RS, Kawalekar OU, Ghassemi S, Milone MC (2018) CAR T cell immunotherapy for human cancer. Science 359:1361-1365. https://doi.org/10.1126/science.aar6711

Kanton S et al (2019) Organoid single-cell genomic atlas uncovers human-specific features of brain development. Nature 574:418 422. https://doi.org/10.1038/s41586-019-1654-9

Karczewski KJ, Snyder MP (2018) Integrative omics for health and disease. Nat Rev Genet 19:299. https://doi.org/10.1038/nrg.2018.4

Kelsey G, Stegle O, Reik W (2017) Single-cell epigenomics: recording the past and predicting the future. Science 358:69. https://doi. org/10.1126/science.aan6826

Kennedy BK et al (2014) Geroscience: linking aging to chronic disease. Cell 159:709-713. https://doi.org/10.1016/j.cell.2014.10.039

Kim W et al (2018) Targeting mutant KRAS with CRISPR-Cas9 controls tumor growth. Genome Res. https://doi.org/10.1101/ gr.223891.117

Krenkel O et al (2020) Myeloid cells in liver and bone marrow acquire a functionally distinct inflammatory phenotype during obesity-related steatohepatitis. Gut 69:551-563. https:// doi.org/10.1136/gutjnl-2019-318382

Kruitwagen HS et al (2017) Long-term adult feline liver organoid cultures for disease modeling of hepatic steatosis. Stem Cell Rep 8:822-830. https://doi.org/10.1016/j.stemcr.2017.02.015

Kuboki Y et al (2019) Single-cell sequencing defines genetic heterogeneity in pancreatic cancer precursor lesions. J Pathol 247:347-356. https://doi.org/10.1002/path.5194

Lake BB et al (2019) A single-nucleus RNA-sequencing pipeline to decipher the molecular anatomy and pathophysiology of human kidneys. Nat Commun 10:2832. https://doi.org/10.1038/ s41467-019-10861-2

Lancaster MA, Huch M (2019) Disease modelling in human organoids. Dis Models Mech 12:dmm09347. https://doi. org/10.1242/dmm.039347

Lareau CA et al (2019) Droplet-based combinatorial indexing for massive-scale single-cell chromatin accessibility. Nat Biotechnol 37:916-924. https://doi.org/10.1038/s41587-019-0147-6

Lawlor N et al (2017a) Single-cell transcriptomes identify human islet cell signatures and reveal cell-type-specific expression changes in type 2 diabetes. Genome Res 27:208-222. https:// doi.org/10.1101/gr.212720.116

Lawlor N, Khetan S, Ucar D, Stitzel ML (2017b) Genomics of islet (Dys)function and type 2 diabetes. Trends Genet 33:244-255. https://doi.org/10.1016/j.tig.2017.01.010

Lee JH et al (2017) Anatomically and functionally distinct lung mesenchymal populations marked by Lgr5 and Lgr6. Cell 170:1149-1163. https://doi.org/10.1016/j.cell.2017.07.028

Levitin HM, Yuan J, Sims PA (2018) Single-cell transcriptomic analysis of tumor heterogeneity. Trends Cancer 4:264-268. https://doi.org/10.1016/j.trecan.2018.02.003

Liao J et al (2020) Single-cell RNA sequencing of human kidney. Sci Data 7:4. https://doi.org/10.1038/s41597-019-0351-8

López-Otín C, Blasco MA, Partridge L, Serrano M, Kroemer G (2013) The hallmarks of aging. Cell 153:1194-1217. https:// doi.org/10.1016/j.cell.2013.05.039

Luecken MD, Theis FJ (2019) Current best practices in single-cell RNA-seq analysis: a tutorial. Mol Syst Biol 15:e8746. https:// doi.org/10.15252/msb.20188746

Ma L, Zheng J (2018) Single-cell gene expression analysis reveals $\beta$-cell dysfunction and deficit mechanisms in type 2 diabetes. BMC Bioinform 19:515. https://doi.org/10.1186/s1285 9-018-2519-1

Macaulay IC, Ponting CP, Voet T (2017) Single-cell multiomics: multiple measurements from single cells. Trends Genet 33:155-168. https://doi.org/10.1016/j.tig.2016.12.003 
MacParland SA et al (2018) Single cell RNA sequencing of human liver reveals distinct intrahepatic macrophage populations. Nat Commun 9:4383. https://doi.org/10.1038/s41467-018-06318-7

Martinez-Jimenez CP et al (2017) Aging increases cell-to-cell transcriptional variability upon immune stimulation. Science 355:1433-1436. https://doi.org/10.1126/science.aah4115

Masoud V, Pagès G (2017) Targeted therapies in breast cancer: New challenges to fight against resistance World. J Clin Oncol 8:120-134. https://doi.org/10.5306/wjco.v8.i2.120

Mincarelli L, Lister A, Lipscombe J, Macaulay IC (2018) Defining cell identity with single-cell omics. Proteomics 18:e1700312e1700312. https://doi.org/10.1002/pmic.201700312

Mithal A et al (2020) Generation of mesenchyme free intestinal organoids from human induced pluripotent stem cells. Nat Commun 11:215. https://doi.org/10.1038/s41467-019-13916-6

Morris SA (2019) The evolving concept of cell identity in the single cell era. Development 146:169748. https://doi.org/10.1242/ dev. 169748

Nagano T et al (2017) Cell-cycle dynamics of chromosomal organization at single-cell resolution. Nature 547:61-67. https://doi. org/10.1038/nature23001

O’Sullivan ED, Hughes J, Ferenbach DA (2017) Renal aging: causes and consequences. J Am Soc Nephrol 28:407. https://doi. org/10.1681/ASN.2015121308

Oetjen KA et al (2018) Human bone marrow assessment by singlecell RNA sequencing, mass cytometry, and flow cytometry. JCI Insight 3:e124928. https://doi.org/10.1172/jci.insight. 124928

Ogrodnik M et al (2017) Cellular senescence drives age-dependent hepatic steatosis. Nat Commun 8:15691. https://doi.org/10.1038/ ncomms 15691

Ouchi R et al (2019) Modeling steatohepatitis in humans with pluripotent stem cell-derived organoids. Cell Metab 30:374-384. https ://doi.org/10.1016/j.cmet.2019.05.007

Park J, Liu C, Kim J, Susztak K (2019) Understanding the kidney one cell at a time. Kidney Int 96:862-870. https://doi.org/10.1016/j. kint.2019.03.035

Park J et al (2018) Single-cell transcriptomics of the mouse kidney reveals potential cellular targets of kidney disease. Science 360:758. https://doi.org/10.1126/science.aar2131

Pepe-Mooney BJ et al (2019) Single-cell analysis of the liver epithelium reveals dynamic heterogeneity and an essential role for YAP in homeostasis and regeneration. Cell Stem Cell 25:23-38. https ://doi.org/10.1016/j.stem.2019.04.004

Podrini C, Borghesan M, Greco A, Pazienza V, Mazzoccoli G, Vinciguerra M (2013) Redox homeostasis and epigenetics in nonalcoholic fatty liver disease (NAFLD). Curr Pharm Des 19:27372746. https://doi.org/10.2174/1381612811319150009

Qian M, Wang DC, Chen H, Cheng Y (2017) Detection of single cell heterogeneity in cancer. Semin Cell Dev Biol 64:143-149. https ://doi.org/10.1016/j.semcdb.2016.09.003

Radpour R, Forouharkhou F (2018) Single-cell analysis of tumors: creating new value for molecular biomarker discovery of cancer stem cells and tumor-infiltrating immune cells. World J Stem Cells 10:160-171. https://doi.org/10.4252/wjsc.v10.i11.160

Rai V et al. (2019) Single cell ATAC-seq in human pancreatic islets and deep learning upscaling of rare cells reveals cell-specific type 2 diabetes regulatory signatures. bioRxiv:749283. https:// doi.org/10.1101/749283

Ramachandran P et al (2019) Resolving the fibrotic niche of human liver cirrhosis at single-cell level. Nature 575:512-518. https:// doi.org/10.1038/s41586-019-1631-3

Regev A et al (2017) The Human Cell Atlas. Elife 6:e27041. https:// doi.org/10.7554/eLife.27041

Rexer BN, Engelman JA, Arteaga CL (2009) Overcoming resistance to tyrosine kinase inhibitors: lessons learned from cancer cells treated with EGFR antagonists. Cell Cycle 8:18-22. https://doi. org/10.4161/cc.8.1.7324

Roerink SF et al (2018) Intra-tumour diversification in colorectal cancer at the single-cell level. Nature 556:457-462. https://doi. org/10.1038/s41586-018-0024-3

Román M, Baraibar I, López I, Nadal E, Rolfo C, Vicent S, Gil-Bazo I (2018) KRAS oncogene in non-small cell lung cancer: clinical perspectives on the treatment of an old target. Mol Cancer 17:33-33. https://doi.org/10.1186/s12943-018-0789-x

Rowland J et al (2019) Uncovering genetic mechanisms of kidney aging through transcriptomics, genomics, and epigenomics. Kidney Int 95:624-635. https://doi.org/10.1016/j.kint.2018.10.029

Rowland J, Akbarov A, Maan A, Eales J, Dormer J, Tomaszewski M (2018) Tick-tock chimes the kidney clock-from biology of renal ageing to clinical applications kidney and blood pressure. Research 43:55-67. https://doi.org/10.1159/000486907

Rozenblatt-Rosen O, Stubbington MJT, Regev A, Teichmann SA (2017) The Human Cell Atlas: from vision to reality. Nature 550:451-453. https://doi.org/10.1038/550451a

Rye IH et al (2018) Intratumor heterogeneity defines treatmentresistant HER2+ breast tumors. Mol Oncol 12:1838-1855. https://doi.org/10.1002/1878-0261.12375

Sachs N et al (2019) Long-term expanding human airway organoids for disease modeling. EMBO J 38:e100300. https://doi. org/10.15252/embj.2018100300

Sant GR, Knopf KB, Albala DM (2017) Live-single-cell phenotypic cancer biomarkers-future role in precision oncology? NPJ Precis Oncol 1:21. https://doi.org/10.1038/s41698-017-0025-y

Segerstolpe $\AA$ et al (2016) Single-cell transcriptome profiling of human pancreatic islets in health and type 2 diabetes. Cell Metab 24:593-607. https://doi.org/10.1016/j.cmet.2016.08.020

Shalek AK, Benson M (2017) Single-cell analyses to tailor treatments. Sci Transl Med 9:eaan4730. https://doi.org/10.1126/ scitranslmed.aan4730

Sierant MC, Choi J (2018) Single-Cell Ssequencing in cancer: recent applications to immunogenomics and multi-omics tools. Genomics Inform 16:e17. https://doi.org/10.5808/ GI.2018.16.4.e17

Song Y, Xu X, Wang W, Tian T, Zhu Z, Yang C (2019) Single cell transcriptomics: moving towards multi-omics. Analyst 144:31723189. https://doi.org/10.1039/c8an01852a

Strzelecka PM, Ranzoni AM, Cvejic A (2018) Dissecting human disease with single-cell omics: application in model systems and in the clinic. Dis Models Mech 11:36525. https://doi.org/10.1242/ dmm.036525

Stuart T, Satija R (2019) Integrative single-cell analysis. Nat Rev Genet 20:257-272. https://doi.org/10.1038/s41576-019-0093-7

Su X et al (2017) Single-cell RNA-Seq analysis reveals dynamic trajectories during mouse liver development. BMC Genomics 18:946. https://doi.org/10.1186/s12864-017-4342-x

Sullivan I, Planchard D (2016) Next-generation EGFR tyrosine kinase inhibitors for treating EGFR-mutant lung cancer beyond first line. Front Med (Lausanne) 3:76. https://doi.org/10.3389/ fmed.2016.00076

Sun YV, Hu Y-J (2016) Integrative analysis of multi-omics data for discovery and functional studies of complex human diseases. In: Friedmann T, Dunlap JC, Goodwin SF (eds) Advances in genetics, vol 93. Academic Press, Cambridge, pp 147-190

Suvà ML, Tirosh I (2019) Single-cell RNA sequencing in cancer: lessons learned and emerging challenges. Mol Cell 75:7-12. https ://doi.org/10.1016/j.molcel.2019.05.003

Svensson V, Vento-Tormo R, Teichmann SA (2018) Exponential scaling of single-cell RNA-seq in the past decade. Nat Protoc 13:599. https://doi.org/10.1038/nprot.2017.149 
Tabula Muris C et al (2018) Single-cell transcriptomics of 20 mouse organs creates a Tabula Muris. Nature 562:367-372. https://doi. org/10.1038/s41586-018-0590-4

Tanaka Y, Cakir B, Xiang Y, Sullivan GJ, Park I-H (2020) Synthetic analyses of single-cell transcriptomes from multiple brain organoids and fetal brain. Cell Rep 30:1682-1689. https://doi. org/10.1016/j.celrep.2020.01.038

Tang X, Huang Y, Lei J, Luo H, Zhu X (2019) The single-cell sequencing: new developments and medical applications. Cell Biosci 9:53. https://doi.org/10.1186/s13578-019-0314-y

Teo AKK et al (2018) Single-cell analyses of human islet cells reveal de-differentiation signatures. Cell Death Discov 4:14. https://doi. org/10.1038/s41420-017-0014-5

Theis FJ, Lickert H (2019) A map of beta-cell differentiation pathways supports cell therapies for diabetes. Nature 569:342-343. https:// doi.org/10.1038/d41586-019-01211-9

Tirosh I, Suvà ML (2019) Deciphering human tumor biology by singlecell expression profiling. Annu Rev Cancer Biol 3:151-166. https ://doi.org/10.1146/annurev-cancerbio-030518-055609

Townsend MH, Shrestha G, Robison RA, O'Neill KL (2018) The expansion of targetable biomarkers for CAR T cell therapy. $\mathrm{J}$ Exp Clin Cancer Res 37:163. https://doi.org/10.1186/s1304 6-018-0817-0

Trapnell C (2015) Defining cell types and states with single-cell genomics. Genome Res 25:1491-1498. https://doi.org/10.1101/ gr.190595.115

Treutlein B et al (2014) Reconstructing lineage hierarchies of the distal lung epithelium using single-cell RNA-seq. Nature 509:371-375. https://doi.org/10.1038/nature13173

Tritschler S, Büttner M, Fischer DS, Lange M, Bergen V, Lickert H, Theis FJ (2019) Concepts and limitations for learning developmental trajectories from single cell genomics. Development 146:dev170506. https://doi.org/10.1242/dev.170506

Tritschler S, Theis FJ, Lickert H, Böttcher A (2017) Systematic single-cell analysis provides new insights into heterogeneity and plasticity of the pancreas. Mol Metab 6:974-990. https://doi. org/10.1016/j.molmet.2017.06.021

Velten L et al (2017) Human haematopoietic stem cell lineage commitment is a continuous process. Nat Cell Biol 19:271-281. https:// doi.org/10.1038/ncb3493

Wagner A, Regev A, Yosef N (2016) Revealing the vectors of cellular identity with single-cell genomics. Nat Biotechnol 34:11451160. https://doi.org/10.1038/nbt.3711

Wagner $\mathbf{J}$ et al (2019) A single-cell atlas of the tumor and immune ecosystem of human. Breast Cancer Cell 177:1330-1345.e1318. https://doi.org/10.1016/j.cell.2019.03.005
Wang J, Song Y (2017) Single cell sequencing: a distinct new field. Clin Transl Med 6:10-10. https://doi.org/10.1186/s4016 9-017-0139-4

Wang J, Xu R, Yuan H, Zhang Y, Cheng S (2019a) Single-cell RNA sequencing reveals novel gene expression signatures of trastuzumab treatment in HER2+ breast cancer: a pilot study. Medicine (Baltimore) 98:e15872. https://doi.org/10.1097/md.0000000000 015872

Wang Q, Guldner IH, Golomb SM, Sun L, Harris JA, Lu X, Zhang S (2019b) Single-cell profiling guided combinatorial immunotherapy for fast-evolving CDK4/6 inhibitor-resistant HER2-positive breast cancer. Nat Commun 10:3817. https://doi.org/10.1038/ s41467-019-11729-1

Wang S et al (2019c) Human ESC-derived expandable hepatic organoids enable therapeutic liver repopulation and pathophysiological modeling of alcoholic liver injury. Cell Res 29:1009-1026. https://doi.org/10.1038/s41422-019-0242-8

Wang W, Gao D, Wang X (2018) Can single-cell RNA sequencing crack the mystery of cells? Cell Biol Toxicol 34:1-6. https://doi. org/10.1007/s10565-017-9404-y

WHO (2009) 2008-2013 Action plan for the global strategy for the prevention and control of noncommunicable diseases, vol 97. World Health Organization, Geneva

Xin $Y$ et al (2016) RNA sequencing of single human islet cells reveals type 2 diabetes genes. Cell Metab 24:608-615. https://doi. org/10.1016/j.cmet.2016.08.018

Xiong X et al (2019) Landscape of intercellular crosstalk in healthy and NASH liver revealed by single-cell secretome gene analysis. Mol Cell 75:644-660. https://doi.org/10.1016/j.molcel.2019.07.028

Zeggini E, Gloyn AL, Barton AC, Wain LV (2019) Translational genomics and precision medicine: moving from the lab to the clinic. Science 365:1409. https://doi.org/10.1126/science.aax45 88

Zhang M et al (2019) Accurate classification of non-small cell lung cancer (NSCLC) pathology and mapping of EGFR mutation spatial distribution by ambient mass spectrometry imaging. Front Oncol. https://doi.org/10.3389/fonc.2019.00804

Publisher's Note Springer Nature remains neutral with regard to jurisdictional claims in published maps and institutional affiliations. 